\title{
APS COMO INSTRUMENTO DE SENSIBILIZACIÓN HACIA LA DISCAPACIDAD EN EDUCACIÓN SUPERIOR
}

\author{
Laura Jiménez Monteagudo \\ Universidad Católica de Valencia \\ S. Vicente Mártir (Valencia) \\ laura.jimenez@ucv.es
}

Recepción Artículo: 13 marzo 2020

Admisión Evaluación: 30 marzo 2020

Informe Evaluador 1: 15 abril2020

Informe Evaluador 2: 19 abril 2020

Aprobación Publicación: 20 abril 2020

\section{RESUMEN}

El Aprendizaje por servicio (APS) es una herramienta utilizada en la educación superior, con la finalidad de enriquecer el aprendizaje y fortalecer las comunidades, siendo fundamental su potencial transformador como movimiento educativo y social (Calvo, Sotelino y Rodriguez, 2019). El APS es un modelo educativo en clave de solidaridad, ya que promueve los valores cívicos y una cultura emprendedora, favoreciendo una participación ciudadana responsable en la atención a las necesidades de la comunidad (Mendía, 2012). El objetivo del estudio fue conocer el impacto de la metodología de APS como medio de sensibilización de los estudiantes del grado de Ciencias de la Actividad Física y el Deporte (CAFD). Se recopilaron y analizaron algunas de las experiencias de APS desarrolladas por los estudiantes de Deporte Adaptado de tercer curso del Grado de CAFD. Se utilizó la entrevista grupal como técnica cualitativa de recogida de información con 8 participantes de los distintos proyectos analizados. Se pudo observar como elemento común que en todas las experiencias hubo contacto directo con personas con discapacidad. Algunas de las experiencias recogidas de los estudiantes mostraron que este tipo de proyectos promovieron una mayor implicación de éstos en la asignatura de Deporte adaptado. Los estudiantes destacaron la metodología del APS como clave para tener experiencias directas con personas con discapacidad y así promover la sensibilización. Sería deseable promover el uso de la metodología de APS en asignaturas como el Deporte Adaptado, en la educación superior, ya que puede ser un instrumento adecuado para sensibilizar a los estudiantes hacia las personas con algún tipo de discapacidad, algo cada vez más necesario para los futuros profesionales del ámbito.

Palabras clave: aprendizaje por servicio; sensibilización; discapacidad; educación superior

\section{ABSTRACT}

Aps as an awareness raising tool towards disability in higher education. Service Learning (SLA) is a tool used in higher education, with the aim of enriching learning and strengthening communities, with its 


\section{APS COMO INSTRUMENTO DE SENSIBILIZACIÓN HACIA LA DISCAPACIDAD EN EDUCACIÓN SUPERIOR}

transformative potential as an educational and social movement being fundamental (Calvo, Sotelino and Rodríguez, 2019). PHC is an educational model based on solidarity, since it promotes civic values and an entrepreneurial culture, encouraging responsible citizen participation in meeting the needs of the community (Mendía, 2012). The objective of the study was to know the impact of the APS methodology as a means of raising awareness among students of the Physical Activity and Sport Sciences degree (CAFD). Some of the PSA experiences developed by the third year Adapted Sport students of the CAFD degree were collected and analyzed. The group interview was used as a qualitative information gathering technique with 8 participants from the different projects analyzed. It could be observed as a common element that in all the experiences there was direct contact with people with disabilities. Some of the experiences collected from the students showed that this type of projects promoted a greater involvement of the students in the subject of adapted sport. The students highlighted the APS methodology as a key to having direct experiences with people with disabilities and thus promoting awareness. It would be desirable to promote the use of the APS methodology in subjects such as Adapted Sport, in higher education, as it can be an adequate instrument to sensitize students towards people with some kind of disability, something that is increasingly necessary for future professionals in the field.

Keywords: service learning; awareness; disability; higher education

\section{INTRODUCCIÓN}

En una sociedad en la que prima el individualismo y los intereses personales, aparecen metodologías solidarias como el Aprendizaje por servicio (en adelante APS), que pretenden dar respuesta a ciertas necesidades dentro del contexto educativo. El ámbito universitario, no queda exento de esta realidad, todo lo contrario, es justamente en este nivel educativo donde parece incluso más necesaria la introducción de metodologías que permitan el compromiso social y la implicación real en proyectos que activen y den sentido al proceso de aprendizaje superior. Así, autores como Vázquez-Rodríguez (2019), resaltan que el Espacio Europeo de Educación Superior (EEES) y los consecuentes procesos de innovación y responsabilidad social derivados del mismo, sitúan al aprendizaje servicio como una herramienta idónea en tal coyuntura.

Algunos de los destacados autores que han impulsado esta metodología de APS la definen como "una propuesta educativa que combina procesos de aprendizaje y de servicio a la comunidad en un único proyecto bien articulado en el que los participantes aprenden a la vez que trabajan en necesidades reales del entorno con la finalidad de mejorarlo" (Puig, Batlle, Bosch y Palos, 2007, p. 20). Además, según Sotelino, Santos-Rego, y Lorenzo (2016), en su trabajo contextualizado en el ámbito universitario, señalan que el aprendizaje servicio es una metodología experiencial que aúna aprendizaje y servicio comunitario en un único proyecto con base cívica y académica. En relación a esta metodología, autores como Mendía (2012), destacan algunas de sus características positivas que pueden ayudarnos a comprender por qué puede ser valiosa en nuestro contexto, la educación superior:

- El aprendizaje mejora el servicio y el servicio mejora el aprendizaje.

- Se crea un proyecto educativo de utilidad social.

- Promueve los valores, como la conciencia cívica y la ética.

- Modelo socioeducativo que promueve actividades solidarias.

En el caso de autores como Calvo, Sotelino y Rodriguez, (2019), nos muestran que el APS proporciona un plus de calidad al aunar en un único proyecto el aprendizaje de contenidos el servicio a la comunidad y el hecho de poder realizarlo también en horario académico, distinguiéndose así de otras propuestas de voluntariado. Además, en relación al ámbito universitario, destacan que el APS es una herramienta utilizada en la educación superior, con la finalidad de enriquecer el aprendizaje y fortalecer las comunidades, siendo fundamental su potencial transformador como movimiento educativo y social. De fondo, y también como característica de esta metodología, destacamos la necesidad de que los estudiantes universitarios se formen en valores que fomenten la inclusión, para así conseguir futuros profesionales del ámbito de la actividad física y el deporte que sean capaces de incluir en sus contextos a personas con cualquier tipo de discapacidad (Mendía, 2016; Ríos, 2012). 
Es este trabajo, trataremos de mostrar los distintos proyectos de APS desarrollados dentro de la asignatura de Deporte Adaptado del grado de Ciencias de la Actividad Física y el deporte de la Universidad Católica de Valencia (UCV). Además, como objetivo principal del trabajo, nos propusimos conocer el impacto de la metodología de APS como medio de sensibilización de los estudiantes del grado de Ciencias de la Actividad Física y el Deporte (CAFD).

\section{METODOLOGÍA E INSTRUMENTOS UTILIZADOS}

Como instrumento de investigación se consideró como más adecuado, la utilización de la entrevista grupal, ya que para este estudio, la entrevista cualitativa, no es meramente un proceso de obtención de información, sino que es un proceso de interacción social (Corbetta, 2007). Desde el criterio de que estuvieran representados todos los proyectos de APS puestos en práctica, se seleccionaron para el estudio 8 estudiantes del tercer curso del grado de Ciencias de la Actividad Física y el Deporte de los distintos proyectos de la asignatura de Deporte Adaptado. De los cuales 6 fueros hombres y 2 mujeres. Todos ellos cumplimentaron el consentimiento informado antes de realizar la entrevista grupal, además de mantenerse su privacidad de datos, cumpliendo así con los requisitos éticos necesarios para llevar a cabo este estudio. La entrevista giró en torno a tres temas fundamentales:

Figura 1 Esquema de temas de la entrevista grupal para el análisis de resultados

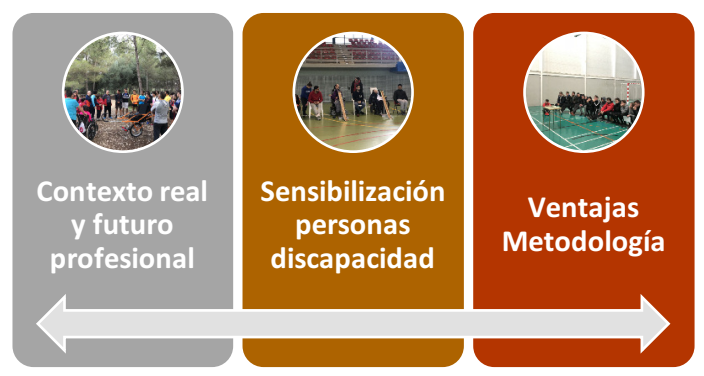

Veamos a continuación los resultados obtenidos en este estudio, tanto de los proyectos realizados como la información de la entrevista grupal.

\section{RESULTADOS Y DISCUSIÓN}

Hemos dividido los resultados en dos apartados diferenciados. En primer lugar y dando respuesta al primer objetivo, describiremos brevemente los cuatro proyectos de APS desarrollados por los estudiantes en la asignatura de Deporte Adaptado. Seguidamente, mostraremos los resultados obtenidos de la entrevista grupal a los participantes.

\section{Proyectos de APS desarrollados}

Tal y como podemos observar en la Figura 2, uno de los grupos de estudiantes realizó su proyecto en un Club deportivo de Boccia. La boccia es un deporte similar a la petanca, que nace para ser practicado por deportistas con discapacidades físicas severas (todos deben ser usuarios de silla de ruedas), fundamentalmente con parálisis cerebral. Los estudiantes, colaboraron en los entrenamientos del equipo, tanto siendo auxiliares (función de ayudante del deportista), como preparando entrenamientos a los deportistas. 


\section{APS COMO INSTRUMENTO DE SENSIBILIZACIÓN HACIA LA DISCAPACIDAD EN EDUCACIÓN SUPERIOR}

Figura 2 Esquema de proyectos de APS en la asignatura de Deporte Adaptado

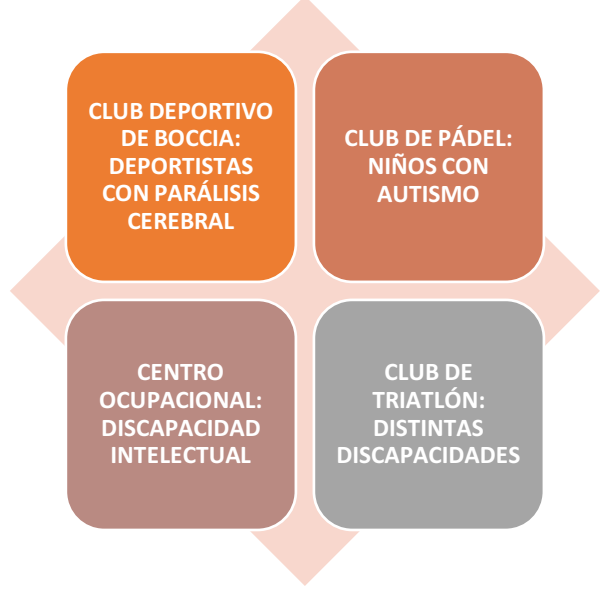

Además, este grupo, diseñó una campaña de publicidad para así poder conseguir personas voluntarias para realizar la función de auxiliares tanto en entrenamientos como en competiciones.

En relación al segundo proyecto, tuvo lugar en un club de pádel en el que los estudiantes, tras realizar el análisis correspondiente de la realidad, planificaron actividades para llevar a cabo con los deportistas con autismo de una forma individualizada.

El tercer proyecto de APS fue el que realizaron los estudiantes en un Centro Ocupacional de la zona. En este caso, la necesidad detectada fue tratar de mejorar las propuestas de calentamiento de las actividades físicas llevadas acabo en el centro para así fomentar la autonomía de los usuarios en la práctica deportiva. Además, se realizó una actividad final con los chicos y chicas del centro ocupacional en las instalaciones de la universidad.

Por último, un grupo de alumnos realizó su proyecto en un club de triatlón con deportistas con diferentes discapacidades. En este caso, la propuesta fue dirigida a la planificación y mejora de los entrenamientos de algunos de los deportistas, tratando así de individualizar el aprendizaje y favorecer así la mejora, tanto de la condición física como de los aspectos técnicos de cada uno.

\section{Resultados entrevista grupal y discusión}

Hemos agrupado los resultados de la entrevista grupal en torno a tres temas fundamentales, por un lado en relación a la experiencia vivida en contextos reales y su influencia en el futuro profesional de los estudiantes, un segundo tema, sobre el impacto de las vivencias en su sensibilización hacia las personas con discapacidad y por último los aspectos que destacaron los participantes sobre la metodología de APS.

Contextos reales y futuro profesional. Cuando los estudiantes fueron preguntados por aspectos a destacar de la experiencia vivida en los proyectos, señalaron la importancia de poder tener un trato personal con personas con discapacidad. Por ejemplo, MM comentó, "Yo pienso que lo que más aprendes, es a tratar con personas con algún tipo de discapacidad". Por su parte, IL resaltó lo importante que había sido realizar este proyecto para conocer más la realidad de las personas con discapacidad, "al no haber tratado nunca con los chicos, al principio cuando íbamos, nos quedábamos un poco más retirados, luego ya cada día te ibas implicando más, ibas aprendiendo a tratar, y ver las necesidades que ellos tenían...". Otro de los aspectos destacables en relación a la importancia de trabajar en contextos reales, fue la referencia que hizo MR a cómo esta experiencia había favorecido su propio conocimiento personal, así como las reacciones ante situaciones reales, algo que se torna fun- 
damental para los futuros profesionales. Lo vemos en comentarios como el de MR, "yo me acercaba, no sabía cómo hablarle, porque no sabía cómo iba a reaccionar, entonces al fin y al cabo yo por ejemplo si no hubiera hecho este trabajo de APS, no sabría actuar...", o el de AL, "entonces aprendes a controlar tus propios sentimientos, y a tener conductas profesionales para sacar lo mejor del deportista... tenga o no discapacidad", en los que vemos cómo estas experiencias pueden favorecer la preparación de nuestros estudiantes para su futuro profesional. Algunos de los autores que han realizado trabajos en el contexto universitario, apoyan estas cuestiones cuando afirman que el APS se presenta como una propuesta educativa clave, que combina el aprendizaje y el servicio a la comunidad, es decir, que conecta la educación formal con el contexto social de proximidad (VázquezRodríguez, 2019). Del mismo modo, Sotelino et al., (2016), recalcan que la universidad es institución educativa y, como tal, debe vincularse a su entorno social inmediato, no debe ser una isla en el extenso océano social, sino que ha de constituir un elemento más del mismo y, por supuesto, uno de sus principales núcleos dinamizadores. Cabe destacar, la aportación de uno de los estudiantes, resaltando que estos proyectos de APS en contextos reales también pueden abrir nuevos campos profesionales que en ocasiones posiblemente el estudiante no los había contemplado como tal. ("conoces a gente también, que luego igual te interesa tener el contacto de esas personas, por si te interesa trabajar en ese ámbito", MM). El estudiante de alguna forma, amplia sus posibilidades de futuro como profesional al conocer de cerca una realidad desconocida hasta el momento.

Sensibilización hacia las personas con discapacidad. En relación al impacto de estas experiencia respecto a la sensibilización de los estudiantes hacia las personas con discapacidad, varios de ellos hicieron alusión a que el contacto directo con personas con discapacidad provocó en ellos un cambio de concepto y de imagen respecto a ellas. Así en las palabras de PC, "El concepto de como tú ves a las personas que tienen discapacidad cambia... e incluso cuando alguien hace algún comentario de alguna persona... le digo .. . oye... y le comento... "(PC). En las palabras del estudiante podemos percibir que no sólo parece haber cambiado su propia imagen respecto a las personas con discapacidad, sino que en su vida cotidiana, ayuda e interviene en ocasiones para que otras personas también puedan cambiarla. También podemos apreciarlo en el comentario de JJ a otro de los estudiantes que no había estado implicado en un proyecto de APS, "Mi compañero estaba súper agobiado, yo le tranquilicé... creo que es vital, muy importante normalizar a las personas con discapacidad, saber que te puedes relacionar con ellos...evitar tantos miedos y prejuicios...". Parece fundamental que los futuros profesionales conciban como prioritaria la necesidad de normalizar la vida de las personas con algún tipo de discapacidad. En la aportación de otro de los participantes, observamos en sus palabras cierta indignación hacia las injusticias en torno a esta realidad y la necesidad de implicarse para poder cambiarla. MM comenta, "Te das cuenta de injusticias y falta de condiciones .... tenían los horarios limitados, el espacio limitado y sobre todo los recursos humanos muy limitados. Ahí te das cuenta de que sí que queda mucho y que nosotros podemos ayudar a que avance." Autores como Puig (2016) enfatizan la importancia de estas vivencias, ya que inciden en que el compromiso tiene que ver con la voluntad de implicarse corporal, mental y emocionalmente en favor de algo que nos ha indignado. En el aprendizaje-servicio, el compromiso se concreta en la participación en la actividad de servicio, en el esfuerzo de aprendizaje para realizar un servicio de calidad y en la voluntad de contribuir con imaginación en todos los momentos del proyecto de aprendizaje-servicio. Así, otro de los estudiantes, valoraba su proyecto ya que expresaba que éste había ayudado a su sensibilización hacia esta realidad, y que no hubiera sido posible sin esta experiencia vivida. En palabras de PC, "Ia sensibilización que hemos tenido nosotros al ir, estar con ellos, trabajar con ellos, es algo que no tienen nuestros compañeros y si no lo hacen no lo van a tener nunca porque oportunidades así, a no ser que tu decidas por tu cuenta o hacer voluntariados o ir a trabajar a un centro de discapacidad que poca gente lo decide no tiene esa vivencia nunca en la vida." Al final, parece enriquecedor dentro del ámbito universitario, que los estudiantes puedan participar e implicarse en cuestiones de alcance social (Vázquez-Rodríguez, 2019), en este caso, en proyectos que faciliten su sensibilización hacia la realidad de las personas con discapacidad. Son muchos los autores que hacen alusión a la necesidad de que los futuros profesionales del ámbito de la actividad física y el deporte reciban formación en relación a la sensibilización hacia las personas con discapacidad para así poder ser impulsores de la inclusión en este ámbito 


\section{APS COMO INSTRUMENTO DE SENSIBILIZACIÓN HACIA LA DISCAPACIDAD EN EDUCACIÓN SUPERIOR}

(Abellán, Sáez-Gallego, Reina, Ferriz y Navarro-Patón, 2019; Block, Hutzler, Barak y Klavina ,2013; JiménezMonteagudo y Hernández-Álvarez, 2013; Reina, 2016).

Ventajas de la metodología de APS. Algunos de los resultados obtenidos respecto a las ventajas del uso de esta metodología de APS, fueron que los estudiantes enfatizaron por unanimidad que esta metodología había reforzado su compromiso, tanto hacia la asignatura de Deporte Adaptado como hacia la realidad social en la que habían actuado. Así, lo vemos en algunos de los comentarios como el de MM, "yo pienso que es en la que más implicación hay por parte de todo el grupo (haciendo referencia a la metodología). Además, al tener el compromiso del club donde estás realizándolo y todo eso, pues te comprometes". En relación a esta implicación de los estudiantes, algunos autores hacen alusión a la necesidad de que los alumnos vayan haciéndose protagonistas de su propio aprendizaje, adquiriendo destrezas y actitudes que nutran de energía emocional su motivación e implicación en tareas y proyectos de carácter curricular y extracurricular (Sotelino et al, 2016).

Otro de los aspectos destacados por los participantes fue que esta metodología al permitir el contacto directo con personas con discapacidad en contextos reales, ayuda a eliminar prejuicios, y por tanto a favorecer el cambio de actitudes. Vemos algunos ejemplos como el que comenta JJ, "El contacto directo ayuda a quitarte prejuicios a romper barreras, a aprender a tratar con normalidad...", o el de OB, "nos haría falta salir más e ir a centros como en este caso... para aprender a tratar en contextos reales...". En el trabajo de Sotelino et al. (2016), los autores dan valor a estas reflexiones de los estudiantes, diciendo que los alumnos que realizan estos proyectos podrán aprender de necesidades que emanan de las propias realidades sociales, gestionando en primera persona la respuesta, algo que puede marcar su trayectoria vital y académica. Por último, otra de las aportaciones hechas por los participantes fue el hecho de valorar esta metodología por considerar que es más efectiva que otras para el aprendizaje de los contenidos de la asignatura. Algunos de los ejemplos que comentaron hacían referencia al aprendizaje de contenidos prácticos de forma más significativa que en las sesiones de la propia asignatura. Una de ellas comentó que "Se aprenden mejor los contenidos. Ya conocíamos la boccia pero practicando allí acabé de entenderlo bien, entonces, también a la hora de los estudios te ayuda mucho más"(MR), algo que afirman algunos autores cuando expresan que estas experiencias favorecen la significatividad, relevancia y utilidad de los aprendizajes, ya que son puestos en acción para clarificar y afrontar problemas básicos de la comunidad y para ampliar conocimientos, sensibilidades y afectos (Mayor y López, 2017). Además, en el trabajo de Gil, Moliner, Bartoll, y García (2016) donde analizaron los beneficios de esta metodología respecto al profesorado de educación física, en diferentes contextos educativos, concluyeron que efectivamente, estos trabajos tienen efectos beneficiosos en la formación de los futuros profesionales.

\section{CONCLUSIONES}

En relación al primer objetivo del estudio, podemos concluir que todos los proyectos de APS desarrollados en la asignatura de Deporte Adaptado fueron llevados a cabo teniendo contacto directo con personas con algún tipo de discapacidad en distintas instituciones o clubes deportivos.

Respecto al impacto de la metodología de APS en los participantes, podemos decir que las experiencias de contacto directo con personas con discapacidad en contextos reales, favorecen el aprendizaje de habilidades tanto en relación al trato con las personas con discapacidad y a dar respuesta a sus necesidades, como en relación al autoconocimiento. La universidad debería acercar a los estudiantes a posibles realidades profesionales, en nuestro caso, parece que la realización de actividades en contextos reales puede ampliar la perspectiva de futuro de los estudiantes, incorporando nuevos ámbitos profesionales como es el ámbito de la actividad física y el deporte con personas con discapacidad, algo que podría ser una consecuencia de la sensibilización hacia esta realidad.

Las experiencias de APS en la asignatura de Deporte Adaptado promovieron el cambio de concepto e imagen respecto a las personas con discapacidad. Además, los estudiantes manifestaron indignación por haber sido conscientes de algunas de las injusticias que tienen que vivir las personas con discapacidad lo que podría ser un signo de sensibilización hacia el ámbito y un impulso para comprometerse en un futuro para cambiar esta realidad social. 
La utilización de la metodología APS acrecentó el compromiso de los estudiantes de Deporte Adaptado tanto hacia la asignatura como hacia la realidad concreta en la que desarrollaron sus proyectos. Parece que la utilización de esta metodología en el ámbito universitario, puede ser clave para fomentar el compromiso de los estudiantes como actitud fundamental en su formación para su futuro profesional.

También podemos concluir, que esta metodología mejoró algunos de los procesos de aprendizaje de los estudiantes en relación a los contenidos de la asignatura, por lo que puede ser una metodología beneficiosa para mejorar estos procesos en el contexto universitario.

Otro de los aspectos positivos del Aprendizaje-servicio realizado, en el que hubo contacto directo con personas con discapacidad, fue que este contacto ayudó a los estudiantes a la eliminación de prejuicios y a romper barreras en relación a este ámbito.

En conclusión, la metodología de APS puede ser una herramienta eficaz para enriquecer la formación inicial de los estudiantes del grado de CAFD, ayudando a acercarlos a la realidad social y así haciendo posible su sensibilización hacia el ámbito de las personas con discapacidad. Esta sensibilización puede ser un impulso para conseguir que los futuros profesionales de la actividad física y el deporte promuevan la inclusión social.

\section{REFERENCIAS BIBLIOGRÁFICAS}

Abellán, J., Sáez-Gallego, N.M., Reina, R., Ferriz, R. y Navarro-Patón, R. (2019) Percepción de autoeficacia hacia la inclusión en futuros maestros de educación física. Revista de Psicología del Deporte/Journal of Sport Psychology, 28 (1) 143-156.

Block, M. Hutzler, Y., Barak, S. y Klavina, A. (2013). Creation and Validation of the Self-Efficacy Instrument for Physical Education Teacher Education Majors Toward Inclusion. Adapted physical activity quarterly. D0I: 10.1123/apaq.30.2.184

Calvo, D., Sotelino, A. y Rodríguez Fernández, J. E. (2019). Aprendizaje-Servicio e inclusión en educación primaria. Una revisión sistemática desde la Educación Física. Retos (36), 611-617.

Corbeta, P. (2007). Metodología y técnicas de investigación social. McGraw-Hill/Interamericana de España, S.A.U.

Gil, J., Moliner, 0., Bartoll, 0., y García, R. (2016). Una experiencia de aprendizaje-servicio en futuros docentes: desarrollo de la competencia social y ciudadana. Revista Complutense de Educación, 27, 53-73.

http://dx.doi.org/10.5209/rev_RCED.2016.v27.n1.45071

Jiménez-Monteagudo, M.L. y Hernández-Álvarez, J.L. (2013). La formación inicial para una Educación Física inclusiva: situación, prospectiva y competencias. Revista Internacional de Medicina y Ciencias de la Actividad Física y el Deporte 13 (51) 471-494. Http://cdeporte.rediris.es/revista/ revista51/artformacion411.htm

Mendía, R. (2016). El aprendizaje-servicio: una metodología para la innovación educativa. Convives, (16), 2-101.

Mendía, R. (2012). El Aprendizaje-Servicio como una estrategia inclusiva para superar las barreras al aprendizaje y a la participación. Revista educación inclusiva, 5 (1).

Mayor Paredes, D. y López Medialdea A. M. (2017). El aprendizaje-servicio como palanca para la inclusión educativa y social. Revista Educação Temas e Problemas, 17 (1-20).

Puig, J. M., Batlle, R., Bosch, C. y Palos, J. (2007). Aprendizaje servicio. Educar para la ciudadanía. Octaedro. Ministerio de EF y Deporte.

Puig, J.M. (2016). Aprendizaje-servicio y educación en valores. Convives, 16, 12-19.

Sotelino, A., Santos-Rego, M.A., y Lorenzo, M. (2016). Aprender y servir en la universidad: Una vía cívica al desarrollo educativo. Teoría de la Educación, 28 (2), 225-248.

Reina, R. (2016). Full inclusion of a student with visual impairment over the full Physical Activity and Sport Sciences Degree: A case study. European Journal of Adapted Physical Activity, 9(1), 40-52

Ríos Hernández, M. (2012). Inclusión del alumnado con discapacidad. Tándem Didáctica de la Educación Física, 38, 7-18. 
Vázquez-Rodríguez, A. (2019). Reseña Guía para la institucionalización del aprendizaje-servicio en la universidad. RIDAS, Revista Iberoamericana de Aprendizaje Servicio, 7, 154-156. D0l10.1344/RIDAS2019.7.11 